\title{
Assessing T-LiDAR technology for high throughput phenotyping apple tree topological and architectural traits.
}

\author{
B. Pallas ${ }^{1, a}$, S. Martinez ${ }^{1}$, O. Simler ${ }^{1}$, E. Carrié ${ }^{1}$, E. Costes $^{1}$ and F. Boudon ${ }^{12}$
}

${ }_{1}^{1}$ UMR AGAP, Univ Montpellier, INRA, CIRAD, Montpellier SupAgro, Montpellier, France; ${ }^{2}$ CIRAD, UMR AGAP, F34398 Montpellier, France

\section{Abstract}

We aim at extending methodologies based on T-Lidar scans for providing a description of individual apple tree topology and architecture.

Experiments were performed on 2- and 3-year-old trees belonging to a core collection of French apple cultivars. In 2016, ten trees were precisely scanned in winter to assess our ability to extract topological traits. In 2017, the whole corecollection was scanned to estimate tree leaf areas at the end of the summer period. Topological reconstructions on 2016 data were performed with Plantscan3D software using existing algorithms whereas an allometric relationship depending on tree alpha hull volumes was used for estimating tree leaf areas on 2017 data. Topological traits extracted from T-LiDAR data were compared to digitizing data of the same trees and the estimated total leaf areas were compared to manual measurements on a sample of twenty trees displaying large variability in vegetative development.

In 2016, topological reconstructions gave promising results with $\mathbf{R}^{2}$ values higher than 0.90 for the total number of axes and growth units pet tree and for their mean length when T-Lidar were compared to digitizing data. However, a significant number of short axes or growth units were not detected by the T-Lidar. Comparisons between T-LiDAR and digitizing data showed good adequacy if axes were classified depending on their branching order suggesting that our method was relevant for evaluating the number of branching points in the structure. Regarding data collected on leafy trees in 2017, the total leaf area estimated from T-LiDAR was also highly correlated $\left(R^{2}=0.83\right)$ with manual measurements.

Forthcoming works are undergoing for improving topological reconstruction algorithms in order to extract topological traits and new variables related to vegetative development on the whole population. Nevertheless this first study suggests that this method could be adapted for phenotypic architectural traits on large tree population on which genetic analyses could be performed.

Keywords: topology, geometry, high throughput phenotypic, structure reconstruction, leaf area.

\footnotetext{
${ }^{a} E-m a i l:$ benoit.pallas@inra.fr
} 